\title{
Overexpression of Long Non-Coding RNA NNT-AS1 Correlates with Tumor Progression and Poor Prognosis in Osteosarcoma
}

\author{
Hui Yea, Jinkuang Lin ${ }^{b} \quad$ Xuedong Yao ${ }^{b}$ Yizhong Lib Xiaobin Lin ${ }^{b}$ Hai Lu \\ aDepartment of Orthopedics, Academy of Orthopedics, The Third Affiliated Hospital of Southern \\ Medical University, Guangdong, 'Department of Orthopedics, The Second Affiliated Hospital of Fujian \\ Medical University, Fujian, China
}

\author{
Key Words \\ Lncrna $\cdot$ NNT-AS1 $\cdot$ Osteosarcoma $・$ Biomarker
}

\begin{abstract}
Background/Aims: Increasing evidence demonstrates that long non-coding RNAs (IncRNAs) play critical regulatory roles in cancers, including osteosarcoma. A previous study showed that Nicotinamide Nucleotide Transhydrogenase-antisense RNA1 (NNT-AS1) was aberrantly expressed in several types of cancer. However, the potential biological roles and regulatory mechanisms of NNT-AS1 in osteosarcoma progression remain unknown. Methods: Quantitative RT-PCR was performed to examine the expression of NNT-AS1 in human tissues and cells. The biological functions of NNT-AS1 were determined by CCK-8, colony formation, Flow cytometry and Transwell assays in vitro. A mouse xenograft model was performed to investigate the effect of NNT-AS1 on tumor growth in vivo. Results: In this study, we found the expression of NNT-AS1 was significantly increased in tumor tissues compared to adjacent normal tissues. Furthermore, upregulated NNT-AS1 expression predicted poor prognosis and was an independent and significant risk factor for osteosarcoma patient survival. Further experiments revealed that NNT-AS1 knockdown significantly inhibited cell proliferation by inducing cell cycle arrest and promoting apoptosis in osteosarcoma cells. Moreover, NNTAS1 silencing suppressed cell migration and invasion in vitro. In a tumor xenograft model, knockdown of NNT-AS1 suppressed tumor growth of OS-732 cells in vivo. Conclusions: Taken together, these findings indicate that NNT-AS1 functions as an oncogene in osteosarcoma and could be a novel diagnostic and therapeutic target for osteosarcoma.
\end{abstract}

Department of Orthopedics, Academy of Orthopedics, Guangdong Province, The Third Affiliated Hospital of Southern Medical University, 183 Zhongshan Avenue West, Guangzhou, 510665, (China); Tel. +86-20-62784240, E-Mail dr_yehui@163.com 


\section{Cellular Physiology Cell Physiol Biochem 2018:45:1904-1914

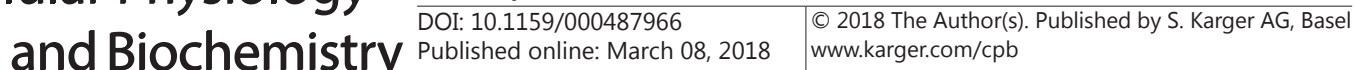

Ye et al.: IncRNA NNT-AS1 Medicates Tumor Growth and Metastasis in Osteosarcoma

\section{Introduction}

Osteosarcoma is the most common primary malignancy of bone and frequently occurs in young adults and adolescents $[1,2]$. With the advancement of radical surgery and neoadjuvant chemotherapy, the survival rates of osteosarcoma patients have dramatically improved in recent decades [3-6]. However, more than $20 \%$ of osteosarcoma patients die from tumor recurrence or metastasis, which remain major obstacles in bone cancer treatment $[3,7]$. Therefore, there is an urgent need to explore the cellular and molecular mechanisms that regulate cancer cell growth and metastasis-related cellular activities.

Long non-coding RNAs (lncRNAs) are a novel class of RNA transcripts, longer than 200 nucleotides in length and with no protein-coding capacity [8, 9]. Increasing evidence indicates that lncRNAs interact with nucleic acids or proteins in diverse biological processes, including cell proliferation, cell differentiation, chromatin remodeling, and drug resistance [9-11]. Previous studies have shown that IncRNAs could act as oncogenes or tumor suppressors during the development and progression of cancers, such as hepatocellular carcinoma, lung cancer, pancreatic cancer, osteosarcoma, and colon cancer [12-17]. For example, IncRNA DGCR5 was decreased in hepatocellular carcinoma tissues and serum, and low DGCR5 expression was associated with HbsAg and vascular invasion. This finding suggests that DGCR5 is an independent negative prognostic factor for hepatocellular carcinoma [12]. HNF1A-antisense 1 (HNF1A-AS1) was highly overexpressed in esophageal adenocarcinoma and promoted tumor growth and metastasis by medicating the expression of cancer-related IncRNA H19 [18]. Recent studies showed that NNT-AS1 was upregulated in colorectal cancer specimens and that knockdown of NNT-AS1 reduced cell proliferation, invasion, induced apoptosis, and inhibited tumor growth and metastasis via inhibiting the epithelial-mesenchymal transition (EMT) and inactivating the MAPK/Erk signaling pathway [19]. Nevertheless, the expression and potential roles of NNT-AS1 during osteosarcoma tumorigenesis and metastasis remain unclear.

In the current study, we investigated the role of NNT-AS1 in osteosarcoma. We found that the expression of NNT-AS1 was remarkably upregulated in osteosarcoma tissues and cell lines, and high NNT-AS1 expression was associated with larger tumors, advanced Enneking stage, metastasis and poor prognosis. Furthermore, we investigated the biological functions of NNT-AS1 in osteosarcoma cell proliferation, migration and invasion in vitro and tumor growth in vivo.

\section{Materials and Methods}

\section{Patients and samples}

A total of 126 freshly frozen OS samples and paired adjacent normal tissue samples were collected from patients who underwent surgical resection between 2007 and 2010 at the Department of Orthopedics in The Third Affiliated Hospital of Southern Medical University (Guangzhou, China). Written informed consent was obtained from all patients who participated in this study. This study was approved by the ethics committee of The Third Affiliated Hospital of Southern Medical University and conducted in accordance with guidelines provided by the Ethics Committees and Institutional Review Boards. Tissue samples were immediately snap-frozen in liquid nitrogen and stored at $-80^{\circ} \mathrm{C}$ until use.

\section{Cell lines and cell cultures}

Immortalized human osteoblast cells (hFOB1.19) and 6 cell lines (U2OS, Saos2, OS-9901, SOSP9607, MG-63 and OS-732) were purchased from the Shanghai Institute of Cell Biology, Chinese Academy of Sciences (Shanghai, China). hFOB1.19 cells were cultured in Dulbecco's minimal essential medium/Ham's F12 (HyClone, Logan, UT, USA). Other human osteosarcoma cells were cultured in Dulbecco's modified Eagle's medium (HyClone) supplemented with 10\% fetal bovine serum (Gibco, Grand Island, NY, USA) at $37^{\circ} \mathrm{C}$ in a $5 \% \mathrm{CO}_{2}$ humidified incubator.

\section{KARGER}




\section{Cellular Physiology Cell Physiol Biochem 2018:45:1904-1914 \begin{tabular}{l|l|l} 
and Biochemistry & $\begin{array}{l}\text { DOI: 10.1159/000487966 } \\
\text { Published online: March 08, } 2018\end{array}$ & $\begin{array}{l}\text { C) } 2018 \text { The Author(s). Published by S. Karger AG, Basel } \\
\text { www.karger.com/cpb }\end{array}$ \\
\hline
\end{tabular}}

\section{Immunohistochemistry}

The dissected tissues from xenograft tumors were fixed in a $10 \%$ formalin solution and processed to obtain paraffin-embedded tissue. After slides were deparaffinized and dehydrated, endogenous peroxidase activity was blocked with $3 \% \mathrm{H}_{2} \mathrm{O}_{2}$ for $10 \mathrm{~min}$ at room temperature. Then, slides were blocked with $10 \%$ normal goat serum for $30 \mathrm{~min}$ and subsequently incubated with antibody against C-myc (Abcam, Cambridge, MA, USA) overnight at $4^{\circ} \mathrm{C}$. After washing with PBS, slides were incubated with biotinylated secondary antibody at room temperature for $1 \mathrm{~h}$. Antigen expression was detected using the DakoCytomation LSAB2 system-HRP (Dako). Slides were counterstained with hematoxylin, dehydrated and mounted. Negative controls were performed by replacing the specific primary antibody with phosphate buffered saline. Evaluation of C-myc staining in tissues was assessed by two independent professional pathologists who were blinded to patient clinical status and outcomes. C-myc expression scores were determined by the area of staining and the percentage of positive cells according to previous work [20].

\section{Reverse transcription and real-time PCR}

Total RNA was extracted from clinical samples and cell lines with TRIzol Reagent (Invitrogen, Carlsbad, CA, USA) according to the manufacturer's protocol. RNA was reverse transcribed using the iScript cDNA Synthesis Kit (Bio-Rad, Hercules, CA, USA). Quantitative PCR was performed with FastStart Universal SYBR Green Master (Roche, Basel, Switzerland) on an ABI7900HT Fast Real-Time PCR System (Applied Biosystems, Foster City, CA, USA). The sequence-specific forward and reverse primers sequences for NNTAS1 were 5'-CTGGAATCCCTGCTACTCAGGA-3' and 5'- GCCATGTGATATGCCTGCTC-3' respectively. Forward and reverse primers sequences for GAPDH were 5'-GTCAACGGATTTGGTCTGTATT-3'; and the reverse primer was 5'-AGTCTTCTGGGTGGCAGTGAT-3' respectively. GAPDH was used as the endogenous control and the relative expression of NNT-AS1 was determined by the $2^{-\Delta \Delta C t}$ method.

\section{Cell proliferation and colony formation assay}

For the cell proliferation assay, $2 \times 10^{3}$ cells in $100 \mu \mathrm{L}$ medium were seeded into a 96-well plate in quadruplicate. $100 \mu \mathrm{L}$ of Cell Counting Kit-8 (CCK-8) (Dojindo; Kumamoto, Japan) was added to each well at 24, 48, 72 and $96 \mathrm{~h}$ after the cells were seeded. The absorbance was measured at a wavelength of $450 \mathrm{~nm}$.

For cell colony analysis, approximately 800 single cells were seeded into each well of a 6-well plate in quadruplicate. Cells were cultured for 2 weeks in a $37^{\circ} \mathrm{C}$ incubator. Cells were washed by PBS and fixed by $4 \%$ paraformaldehyde, stained with $1 \%$ crystal violet for $20 \mathrm{~min}$, and then washed with water. The number of colonies with $>50$ cells was counted manually under an inverted microscope.

\section{Flow cytometry analysis}

For cell apoptosis assays, cells were harvested and gently resuspended in $100 \mu \mathrm{l}$ of binding buffer containing $5 \mu \mathrm{l}$ of FITC Annexin V and $5 \mu \mathrm{l}$ of propidium iodide (PI), and then incubated for $15 \mathrm{~min}$ at room temperature in the dark. The stained cells were analyzed by flow cytometry (BD Biosciences, San Diego, CA, USA).

For cell cycle analysis, cells were harvested and fixed with $70 \%$ cold ethanol at $4^{\circ} \mathrm{C}$ overnight. Then, cells were incubated with PI for $10 \mathrm{~min}$, and the DNA content was determined by flow cytometry.

\section{Cell migration and invasion assays}

Cell migration assays were performed using 24 -well Transwell plates $(8.0-\mu \mathrm{m}$ pore membranes; Costar). Approximately $5 \times 10^{4}$ OS cells transduced with NC or shRNA were plated in the upper chamber in a serum-free medium. The lower chambers were filled with $600 \mu \mathrm{l}$ of medium containing $10 \%$ FBS as a chemoattractant. After a $36 \mathrm{~h}$ incubation, the bottom of the chamber insert was stained with $1 \%$ crystal violet for $20 \mathrm{~min}$. For the cell invasion assay, the procedure was similar to the cell migration assay, except that Transwell membranes were pre-coated with Matrigel (BD Biosciences). The cells that had invaded through the membrane to the lower surface were photographed and counted under an inverted microscope in 6 random fields with a 200x magnification.

Protein extraction and western blot analysis

Cellular protein was lysed in RIPA Lysis Buffer (Cwbiotech, Beijing, China) supplemented with complete protease inhibitor cocktail tablets (Roche, Basel, Switzerland). Equal amounts of protein were separated by 


\section{Cellular Physiology Cell Physiol Biochem 2018;45:1904-1914 \\ \begin{tabular}{l|l} 
and Biochemistry Published onlIne: March 08, 2018 & $\begin{array}{l}\text { (c) } 2018 \text { The Author(s). Published by S. Karger AG, Basel } \\
\text { www.karger.com/cpb }\end{array}$
\end{tabular}}

Ye et al.: IncRNA NNT-AS1 Medicates Tumor Growth and Metastasis in Osteosarcoma

10\% SDS-PAGE and then transferred to PVDF membranes (Bio-Rad). After the membranes were blocked with $5 \%$ non-fat milk at room temperature for $1 \mathrm{~h}$, the membranes were incubated with primary antibodies for AKT (Cell Signal Technology, Danvers, MA, USA), Phospho-Akt (Ser473) (Cell Signal Technology), MMP2 (Cell Signal Technology), MMP-9 (Cell Signal Technology), BCL-2 (Abcam), C-myc (Abcam), CyclinD1 (Abcam) and GAPDH (Cwbiotech) at $4^{\circ} \mathrm{C}$ overnight.

\section{Xenograft Model}

All mouse experiments were performed with the approval of the Animal Studies Ethics Committee of The Third Affiliated Hospital of Southern Medical University according to the Guide for the Care and Use of Laboratory Animals. OS-732 cells $\left(1 \times 10^{7}\right)$ transduced with NC or XIST-shRNA were subcutaneously injected into the right flank of 4-week-old BALB/c nude mice (Shanghai Slaccas Laboratory Animal Company). Tumor length and width were measured every week after injection using calipers. At 5 weeks after injection, the mice were sacrificed and the tumor tissues were collected for further IHC analysis.

\section{Statistical analysis}

Statistical analysis was performed using GraphPad Prism 5 software (GraphPad Software, Inc., La Jolla, CA, USA). Data were presented as the mean \pm standard deviation (SD) from at least three separate experiments. Differences between groups were analyzed using Student's t test and the $\chi 2$ test. Survival curves were plotted using the Kaplan-Meier method and the log-rank test. $\mathrm{P}<0.05$ was considered statistically significant.

\section{Results}

NNT-AS1 expression is increased in osteosarcoma tissues and cell lines

To assess the expression of NNT-AS1 in osteosarcoma tissues, the expression of NNT-AS1 was detected in 126 paired osteosarcoma cancer samples and their corresponding adjacent non-tumor tissues by qRT-PCR. As shown in Fig. 1A and $1 \mathrm{~B}$, the expression level of NNT-AS1 was significantly overexpressed in the primary tumor tissue of patients, while there was no difference in NNT-AS1 expression between benign tissues and healthy controls (Fig. 1B). Meanwhile, the expression of NNT-AS1 was remarkably upregulated in with higher Enneking stage (IIB-III) osteosarcoma tissues compared with lower Enneking stage (IA-IIA) osteosarcoma tissues (Fig. $1 \mathrm{C})$. In addition, we explored the expression of NNT-AS1 in 6 osteosarcoma cells (U20S, Saos2, OS-9901, SOSP-9607, MG-63 and OS-732) and osteoblast cells (hFOB1.19). Our results indicated that NNT-AS1 expression was

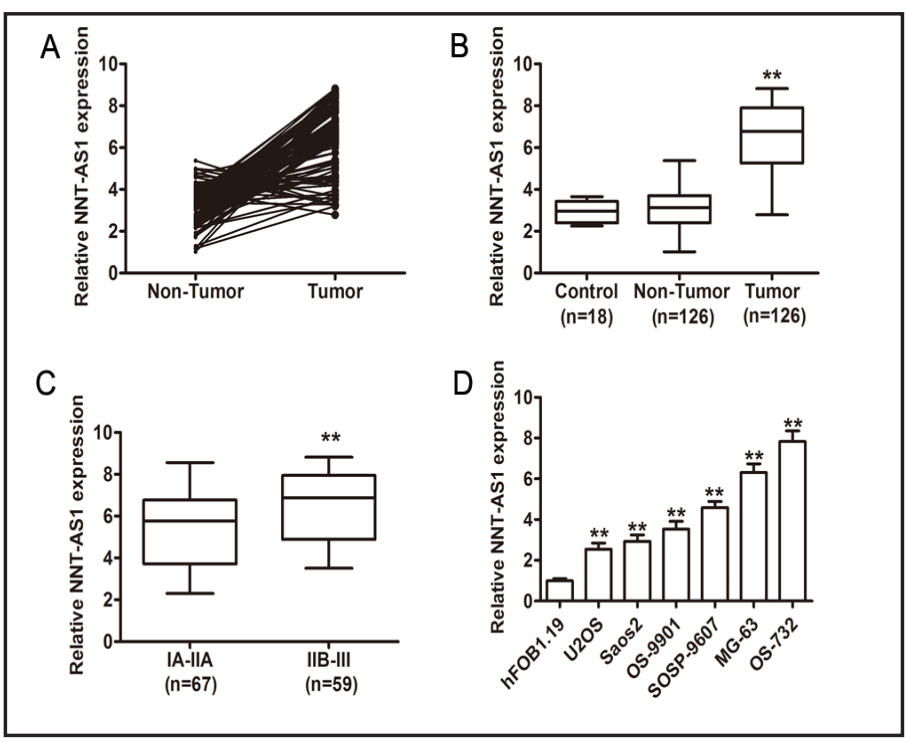

Fig. 1. NNT-AS1 expression is increased in OS tissues. (A) Relative expression of NNT-AS1 in osteosarcoma tissues compared with their adjacent healthy tissues. (B) The expression of NNT-AS1 was significantly upregulated in osteosarcoma samples compared to the adjacent non-malignant tissues. (C) The upregulation of HOTAIR was significantly associated with advanced Enneking stage. (D) qRT-PCR was performed to detect the expression of NNT-AS1 in an osteoblastic cell line (hFOB1.19) and the osteosarcoma cell lines (U20S, Saos2, OS-9901, SOSP-9607, MG-63 and 0S-732). ${ }^{* *} \mathrm{p}<0.01$. 


\section{Cellular Physiology Cell Physiol Biochem 2018;45:1904-1914 and Biochemistry \begin{tabular}{l|l} 
DOI: 10.1159/000487966 & $\begin{array}{l}\text { C } 2018 \text { The Author(s). Published by S. Karger AG, Basel } \\
\text { www.karger.com/cpb }\end{array}$
\end{tabular}

increased in all 6 osteosarcoma cell lines compared with hFOB1.19 (Fig. 1D). These results showed that NNT-AS1 may function as an oncogene in osteosarcoma.

Overexpression of NNT-AS1 is associated with malignant status and overall survival in OS patients

To evaluate the relationship between NNT-AS1 expression and clinicopathological parameters, we divided the 126 patients into high and low NNT-AS1 expression groups based on the median value of NNT-AS1 expression. As shown in Table 1, the NNTAS1 expression level was significantly higher in larger tumors (diameter $>5$ $\mathrm{cm}, \mathrm{P}=0.0333$ ), more advanced Enneking stage tumors $(\mathrm{P}=0.0041)$, and metastasis $(\mathrm{P}=0.0233)$. Nevertheless, no significant relationship was observed between NNT-AS1 expression and other clinical parameters (Table 1). Moreover, KaplanMeier analysis showed that the overall survival rate (Fig. 2A) and disease-free survival rate (Fig. 2B) of the high NNTAS1 expression group was significantly worse than that of the low NNT-AS1 expression group. These results suggest that NNT-AS1 is involved in osteosarcoma aggressiveness and progression.

Knockdown of NNT-AS1 repressed osteosarcoma cell proliferation by affecting cell apoptosis and cell cycle

Since NNT-AS1 expression was associated with tumor size, we explored the biological functions of NNT-AS1 by short hairpin RNA (shRNA)-mediated NNT-AS1 downregulation in osteosarcoma cell lines. Two NNT-AS1-targeted shRNAs (shRNA\#1 and shRNA\#2) could downregulate the expression of NNT-AS1 in both MG-63 and OS-732 cells by qRT-PCR (Fig. 3A). CCK-8 assays indicated that depletion of NNT-AS1 significantly suppressed MG-63 and OS-732 cell proliferation compared with a control group (NC) (Fig. 3B). Data from a colony formation assay also revealed that inhibition of NNT-AS1 caused a substantial reduction in cell survival (Fig. 3C). To investigate potential mechanisms underlying the growth-inhibitory effects of NNT-AS1 knockdown, we assessed apoptosis and cell cycle in MG-63 and OS-732 cells. Compared to the si-NC transfected cells, NNT-AS1 knockdown resulted in a significant increase in MG-63 and OS-732 cell apoptosis (Fig. 3D). Cell cycle analysis suggested that depletion of NNT-AS1 decreased the number of MG-63 and OS-732 cells in S phase compared to cells transfected with si-NC (Fig. 3E). These results indicate that NNT-AS1 is involved in osteosarcoma cell proliferation partially by promoting cell cycle progression and inhibiting cell apoptosis.
Table 1. Correlation between NNT-AS1 expression and clinicopathological parameters of osteosarcoma

\begin{tabular}{lccc}
\hline \multicolumn{3}{c}{ NNT-AS1 expression level } \\
\hline $\begin{array}{l}\text { Characters } \\
\text { Gender }\end{array}$ & Low (N=63) & High (N=63) & P-value \\
Female & 24 & 19 & 0.4525 \\
Male & 39 & 44 & \\
Age, & & & 0.5894 \\
$\leq 20$ & 34 & 38 & \\
$>20$ & 29 & 25 & \\
Tumor size (cm) & & & 0.0333 \\
$<5$ & 40 & 27 & \\
$\geq 5$ & 23 & 36 & 0.0041 \\
Enneking stage & & & \\
IA-IIA & 42 & 25 & 0.8346 \\
IIB-III & 21 & 38 & \\
pathologic fracture & & & \\
No & 49 & 47 & \\
Yes & 14 & 16 & \\
Location & & & \\
Femur & 28 & 26 & \\
Tibia & 20 & 18 & \\
Other & 15 & 19 & \\
Metastasis & & & \\
No & 48 & 27 & \\
Yes & 15 & & \\
*Fisher's exact tests, and X & tests for all the other analysis. \\
\hline
\end{tabular}

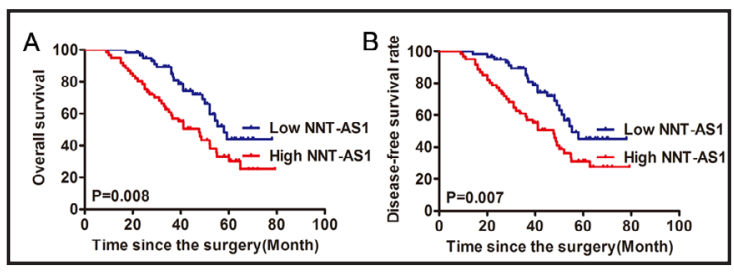

Fig. 2. High NNT-AS1 expression is associated with worse survival in osteosarcoma. (A) The overall survival (OS) of osteosarcoma patients with high NNTAS1 expression was less than that of patients with low NNT-AS1 expression. (B) Kaplan-Meier analysis shows the disease-free survival curves of patients with high NNT-AS1 expression was shorter than that of patients with low NNT-AS1 expression. 


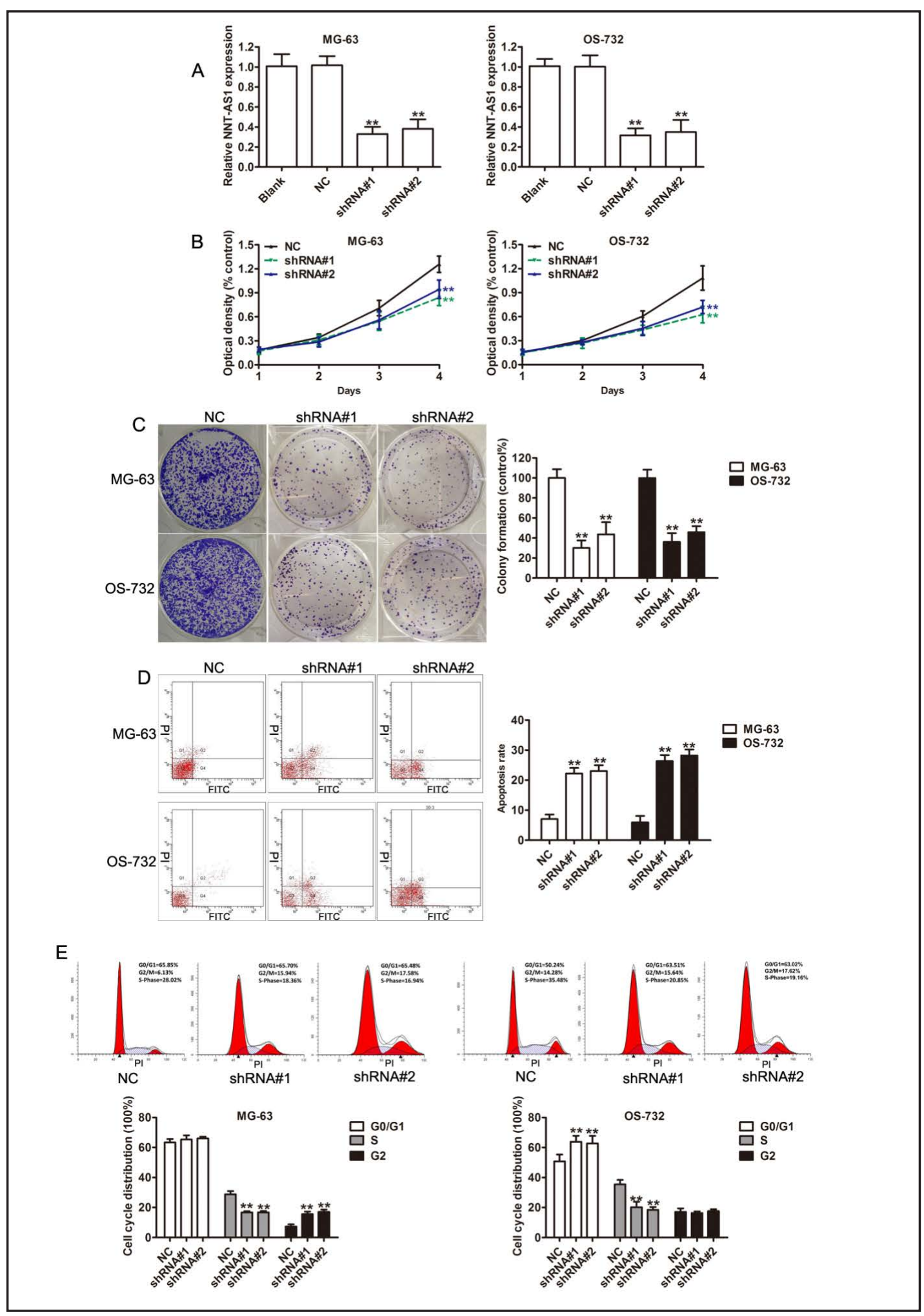

Fig. 3. NNT-AS1 knockdown inhibits cell proliferation in vitro. (A) The expression of NNT-AS1 in MG-63 and OS-732 cells transduced with NC or NNT-AS1 shRNA was measured using qRT-PCR. (B) The CCK-8 assay and (C) colony formation assay were used to evaluate the proliferation of the MG-63 and OS-732 cells after being transduced with NC or NNT-AS1 shRNA. Flow cytometry was performed to assess cell apoptosis (D) and cell cycle progression (E) of MG-63 and OS-732 cells after NNT-AS1 knockdown. 


\section{Cellular Physiology \begin{tabular}{l|l|l} 
and Biochemistry & $\begin{array}{l}\text { DOI: 10.1159/000487966 } \\
\text { Published online: March 08, } 2018\end{array}$ & $\begin{array}{l}\text { C) 2018 The Author(s). Published by S. Karger AG, Basel } \\
\text { www.karger.com/cpb }\end{array}$ \\
\hline
\end{tabular}}

Knockdown of NNT-AS1 suppressed osteosarcoma cell migration and invasion

To further assess the effect of NNT-AS1 on the migration and invasion of osteosarcoma cells, Transwell migration assays were performed. Transwell migration assays indicated that NNT-AS1 silencing markedly repressed MG-63 (Fig. 4A) and OS-732 (Fig. 4B) migration and invasion compared to si-NC. Furthermore, based on the above effects of NNT-AS1 on osteosarcoma cells, we detected the expression of AKT, p-AKT, BCL-2, C-myc, CyclinD1, MMP2 and caspase- 9 by western blot. As shown in Fig. 4C, the protein levels of p-AKT, BCL-2, C-myc, CyclinD1, MMP-2 and MMP-9 were significantly decreased in NNT-AS1 knockdown MG-63 and 0S-732 cells (Fig. 4C). These results indicate that NNT-AS1 plays a role in enhancing the invasion ability of osteosarcoma cells.

\section{Stable knockdown of NNT-AS1 inhibited tumor growth in vivo}

To further explore the effects of NNT-AS1 on the growth of osteosarcoma xenografts in nude mice, OS-732 cells stably transfected with shRNA\#1 were inoculated into male nude mice. Five weeks later, in vivo study showed that xenograft tumors formed by the NNT-AS1silenced cells had smaller volumes and weighed less than those formed by NC-treated cells

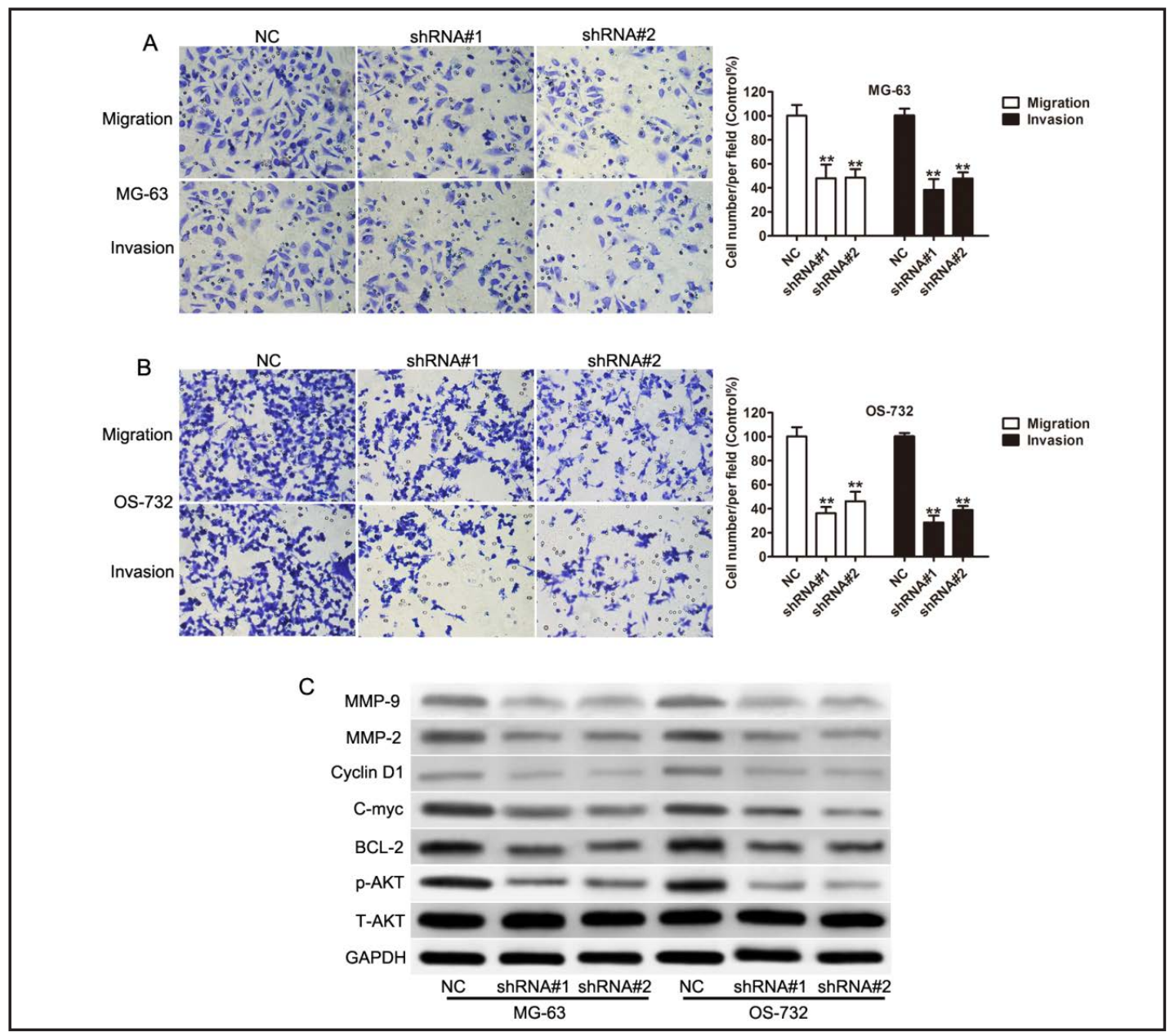

Fig. 4. NNT-AS1 silencing inhibits osteosarcoma cell migration and invasion. (A) Transwell migration and invasion assays were performed to evaluate the invasive ability of MG-63 cells transduced with NC or NNTAS1 shRNA. (B) Transwell migration and invasion assays were performed to evaluate the invasive ability of OS-732 cells transduced with NC or NNT-AS1 shRNA. (C) Western blot analysis of total AKT, p-AKT, BCL-2, C-myc, CyclinD1, MMP-2 and MMP-9 proteins in osteosarcoma cells with or without NNT-AS1 knockdown. $* * \mathrm{p}<0.01$. 


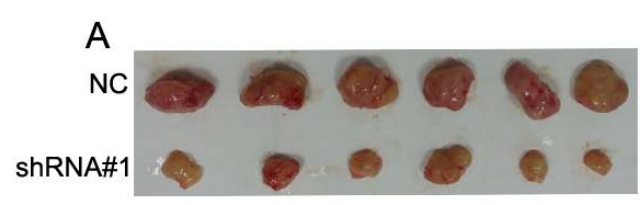

C

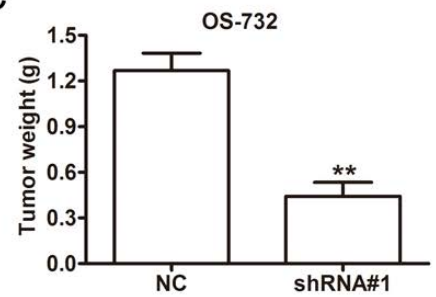

E
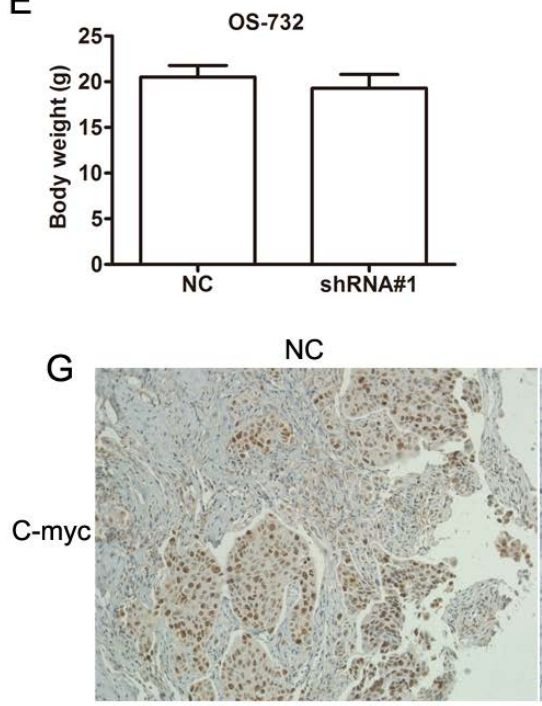

B

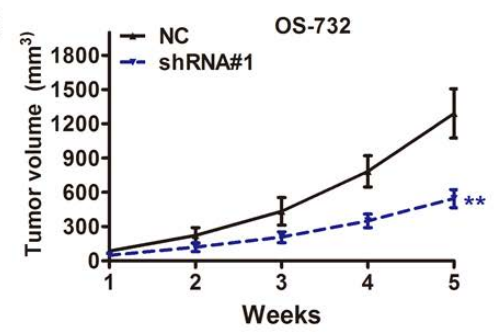

D

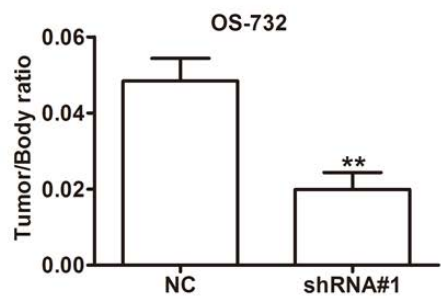

$\mathrm{F}$

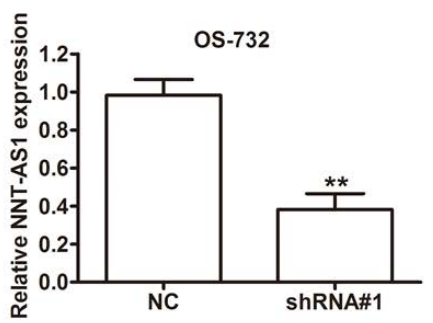

ShRNA\#1

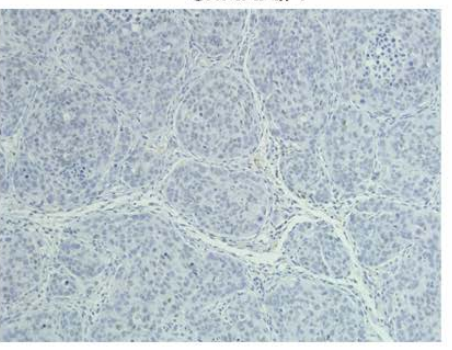

Fig. 5. Inhibition of the growth of OS-732 xenografts by NNT-AS1 knockdown in vivo. (A) Representative xenograft tumors formed by OS-732 cells transduced with NC or NNT-AS1 shRNA. (B) Tumor growth was significantly slower in mice injected with NNT-AS1 knockdown cells. (C) Tumor weights as shown in A. (D) The tumor/body weight ratio. (E) The body weight of BALB/c (nu/nu) mice. (F) The expression of NNTAS1 was measured in xenografts tumors. (G) Immunohistochemical staining with a Ki67 antibody was performed on paraffin-embedded sections of xenografts tumors from 0S-732 cells with or without NNT-AS1 knockdown. ${ }^{* *} \mathrm{p}<0.01$.

(Fig. 5A-D). However, there was no statistically significant difference in body weight between the NC group and NNT-AS1-silenced group (Fig. 5E). qRT-PCR revealed that NNT-AS1 was significantly decreased in tumors from the NNT-AS1-silenced group (Fig. 5F). In addition, the tissues resected from the xenograft tumors showed that the tumors developed from NNT-AS1-silenced cells displayed lower C-myc staining than those of the NC group (Fig. 5G). Taken together, these results are consistent with the in vitro findings, which confirmed the proto-oncogenic role of NNT-AS1 in osteosarcoma tumorigenesis. 


\section{Cellular Physiology Cell Physiol Biochem 2018;45:1904-1914

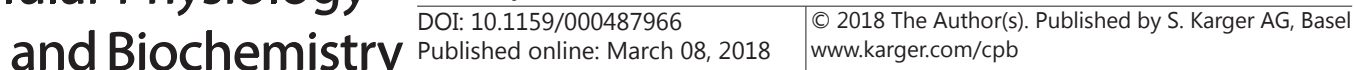 \\ Ye et al.: IncRNA NNT-AS1 Medicates Tumor Growth and Metastasis in Osteosarcoma}

\section{Discussion}

Deregulation of lncRNAs has been shown to contribute to initiation and progression in various human cancer types, including hepatocellular carcinoma, lung cancer, pancreatic cancer, osteosarcoma and colon cancer $[13,15,16,20]$. Even though more and more lncRNAs have been reported to play crucial roles in osteosarcoma [15, 17, 21], the roles of lncRNAs in osteosarcoma are remain poorly defined. The identification of osteosarcoma-associated lncRNAs was needed to better understand the initiation and progression of osteosarcoma.

In this study, we found that NNT-AS1 was obviously upregulated in osteosarcoma tissues and cell lines, compared to the adjacent non-tumor tissues and immortalized human osteoblast cells. Statistical analysis revealed that high NNT-AS1 expression was associated with tumor size, advanced Enneking stage, metastasis and poor prognosis. Thus, we explored the biological function of NNT-AS1 in osteosarcoma by silencing NNT-AS1. Our in vitro shRNA-mediated NNT-AS1 silencing resulted in inhibition of cell proliferation and motility.

Recent study has shown that NNT-AS1 was significantly increased in colorectal cancer tissues and could be used as a biomarker of poor prognosis [19]. Consistent with this previous study, our results revealed that NNT-AS1 was significantly upregulated in osteosarcoma tissues and was positively associated with poor prognosis. These data indicate that NNT-AS1 might act as an oncogene in the development of osteosarcoma. Uncontrolled cell proliferation and metastasis are the major cause of cancer. The shRNA-mediated NNTAS1 silencing reduced CRC cell proliferation and colony formation in vitro. Knockdown of NNT-AS1 mediated CRC cell migration and invasion, which possibly attenuated EMT and the MAPK/Erk signaling pathway [19]. In our present study, NNT-AS1 silencing suppressed cell proliferation, migration and invasion in vitro. In addition, flow cytometry analysis showed that knockdown of NNT-AS1 contributed to a significant increase in cell apoptosis. To further elucidate the regulatory mechanism of NNT-AS1's involvement in cell proliferation, migration and invasion, proteins involved in proliferation, migration and invasion were detected by western blotting. Our results indicated that NNT-AS1 silencing remarkably decreased the expression of phosphorylated levels of AKT, C-myc, BCL-2, MMP-2 and MMP-9. LncRNAs are highly upregulated in liver cancer (HULC) and promote cell proliferation and invasion via the PI3K/Akt/mTOR signaling pathway in glioma [22]. LncRNA AK023948 was found to be a positive regulator of AKT contributing to breast tumor progression [23]. Other studies have reported that IncRNAs medicate tumor progression via CyclinD1, MMP-2 and MMP-9, which are important in cancer development [24, 25]. NNT-AS1 silencing impaired tumor growth in vivo, also confirming that NNT-AS1 acts as a proto-oncogene in osteosarcoma.

\section{Conclusion}

In summary, our study provides the first evidence that the dysfunction of NNT-AS1 expression is associated with the progression of osteosarcoma. We show that inhibition of NNT-AS1 suppresses cell proliferation and tumor growth in vitro and in vivo. Knockdown of NNT-AS1 suppresses migration and invasion abilities in vitro. Other possible biological roles of NNT-AS1 will be explored in future studies.

\section{Acknowledgements}

This study was supported in part by the National Natural Scientific Foundation of China (No 8157100055), the Young People Foundation of Health Department of Fujian Provincial of China (No 2016-ZQN-57), the Fujian Provincial Natural Science Foundation (No.2017J01181) and the Quanzhou Science and Technology Program (No.2016Z46). 


\section{Cellular Physiology Cell Physiol Biochem 2018;45:1904-1914 \begin{tabular}{ll|l} 
and BiOChemistry & $\begin{array}{l}\text { DOI: 10.1159/000487966 } \\
\text { Published online: March 08, 2018 }\end{array}$ & $\begin{array}{l}\text { (c) } 2018 \text { The Author(s). Published by S. Karger AG, Basel } \\
\text { www.karger.com/cpb }\end{array}$
\end{tabular}}

Ye et al.: IncRNA NNT-AS1 Medicates Tumor Growth and Metastasis in Osteosarcoma

\section{Disclosure Statement}

The authors declare that there are no conflicts of interest regarding the publication of this paper.

\section{References}

1 Collins M, Wilhelm M, Conyers R, Herschtal A, Whelan J, Bielack S, Kager L, Kuhne T, Sydes M, Gelderblom H, Ferrari S, Picci P, Smeland S, Eriksson M, Petrilli AS, Bleyer A, Thomas DM: Benefits and adverse events in younger versus older patients receiving neoadjuvant chemotherapy for osteosarcoma: Findings from a meta-analysis. J Clin Oncol 2013;31:2303-2312.

-2 Bielack S, Carrle D, Casali PG: Osteosarcoma: ESMO clinical recommendations for diagnosis, treatment and follow-up. Ann Oncol 2009;20 Suppl 4:137-139.

- 3 Hasei J, Sasaki T, Tazawa H, Osaki S, Yamakawa Y, Kunisada T, Yoshida A, Hashimoto Y, Onishi T, Uno F, Kagawa S, Urata Y, Ozaki T, Fujiwara T: Dual programmed cell death pathways induced by p53 transactivation overcome resistance to oncolytic adenovirus in human osteosarcoma cells. Mol Cancer Ther 2013;12:314-325.

4 Mirabello L, Troisi RJ, Savage SA: Osteosarcoma incidence and survival rates from 1973 to 2004: Data from the Surveillance, Epidemiology, and End Results Program. Cancer 2009;115:1531-1543.

5 Carrle D, Bielack S: Osteosarcoma lung metastases detection and principles of multimodal therapy. Cancer Treat Res 2009;152:165-184.

6 Zhou W, Hao M, Du X, Chen K, Wang G, Yang J: Advances in targeted therapy for osteosarcoma. Discov Med 2014;17:301-307.

7 Sakamoto A, Iwamoto Y: Current status and perspectives regarding the treatment of osteo-sarcoma: Chemotherapy. Rev Recent Clin Trials 2008;3:228-231.

8 Mattick JS: The genetic signatures of noncoding RNAs. PLoS Genet 2009;5:e1000459.

-9 Ulitsky I, Bartel DP: LincRNAs: Genomics, evolution, and mechanisms. Cell 2013;154:26-46.

10 Kung JT, Colognori D, Lee JT: Long noncoding RNAs: Past, present, and future. Genetics 2013;193:651-669.

-11 Mercer TR, Dinger ME, Mattick JS: Long non-coding RNAs: Insights into functions. Nat Rev Genet 2009;10:155-159.

12 Huang R, Wang X, Zhang W, Zhangyuan G, Jin K, Yu W, Xie Y, Xu X, Wang H, Sun B: Down-Regulation of LncRNA DGCR5 correlates with poor prognosis in hepatocellular carcinoma. Cell Physiol Biochem 2016;40:707-715.

13 Chen Y, Li C, Pan Y, Han S, Feng B, Gao Y, Chen J, Zhang K, Wang R, Chen L: The emerging role and promise of long noncoding RNAs in lung cancer treatment. Cell Physiol Biochem 2016;38:2194-2206.

14 Zhou M, Diao Z, Yue X, Chen Y, Zhao H, Cheng L, Sun J: Construction and analysis of dysregulated lncRNAassociated ceRNA network identified novel IncRNA biomarkers for early diagnosis of human pancreatic cancer. Oncotarget 2016;7:56383-56394.

15 Zhao H, Hou W, Tao J, Zhao Y, Wan G, Ma C, Xu H: Upregulation of lncRNA HNF1A-AS1 promotes cell proliferation and metastasis in osteosarcoma through activation of the Wnt/beta-catenin signaling pathway. Am J Transl Res 2016;8:3503-3512.

-16 Li S, Hua Y, Jin J, Wang H, Du M, Zhu L, Chu H, Zhang Z, Wang M: Association of genetic variants in IncRNA H19 with risk of colorectal cancer in a Chinese population. Oncotarget 2016;7:25470-25477.

17 Chen R, Wang G, Zheng Y, Hua Y, Cai Z: Long non-coding RNAs in osteosarcoma. Oncotarget 2017

18 Yang X, Song JH, Cheng Y, Wu W, Bhagat T, Yu Y, Abraham JM, Ibrahim S, Ravich W, Roland BC, Khashab M, Singh VK, Shin EJ, Yang X, Verma AK, Meltzer SJ, Mori Y: Long non-coding RNA HNF1A-AS1 regulates proliferation and migration in oesophageal adenocarcinoma cells. Gut 2014;63:881-890.

-19 Wang Q, Yang L, Hu X, Jiang Y, Hu Y, Liu Z, Liu J, Wen T, Ma Y, An G, Feng G: Upregulated NNT-AS1, a long noncoding RNA, contributes to proliferation and migration of colorectal cancer cells in vitro and in vivo. Oncotarget 2017;8:3441-3453.

20 Wang F, Yang H, Deng Z, Su Y, Fang Q, Yin Z: HOX antisense lincRNA HOXA-AS2 promotes tumorigenesis of hepatocellular carcinoma. Cell Physiol Biochem 2016;40:287-296. 
Ye et al.: IncRNA NNT-AS1 Medicates Tumor Growth and Metastasis in Osteosarcoma

21 Xie CH, Cao YM, Huang Y, Shi QW, Guo JH, Fan ZW, Li JG, Chen BW, Wu BY: Long non-coding RNA TUG1 contributes to tumorigenesis of human osteosarcoma by sponging miR-9-5p and regulating POU2F1 expression. Tumour Biol 2016;37:15031-15041.

22 Zhu Y, Zhang X, Qi L, Cai Y, Yang P, Xuan G, Jiang Y: HULC long noncoding RNA silencing suppresses angiogenesis by regulating ESM-1 via the PI3K/Akt/mTOR signaling pathway in human gliomas. Oncotarget 2016;7:14429-14440.

-23 Koirala P, Huang J, Ho TT, Wu F, Ding X, Mo YY: LncRNA AK023948 is a positive regulator of AKT. Nat Commun 2017;8:14422.

24 Lu X, Zhou C, Li R, Deng Y, Zhao L, Zhai W: Long noncoding RNA AFAP1-AS1 promoted tumor growth and invasion in cholangiocarcinoma. Cell Physiol Biochem 2017;42:222-230.

25 Lv QL, Hu L, Chen SH, Sun B, Fu ML, Qin CZ, Qu Q, Wang GH, He CJ, Zhou HH: A long noncoding RNA ZEB1AS1 promotes tumorigenesis and predicts poor prognosis in glioma. Int J Mol Sci 2016;17 